\title{
Téoros
}

Revue de recherche en tourisme

\section{Le déclin de Floribec}

\section{Rémy Tremblay}

Volume 22, numéro 2, été 2003

URI : https://id.erudit.org/iderudit/1071598ar

DOI : https://doi.org/10.7202/1071598ar

Aller au sommaire du numéro

\section{Éditeur(s)}

Université du Québec à Montréal

\section{ISSN}

0712-8657 (imprimé)

1923-2705 (numérique)

Découvrir la revue

\section{Citer cet article}

Tremblay, R. (2003). Le déclin de Floribec. Téoros, 22(2), 63-66. https://doi.org/10.7202/1071598ar d'utilisation que vous pouvez consulter en ligne.

https://apropos.erudit.org/fr/usagers/politique-dutilisation/ 


\section{Le déclin de Floribec'}

\section{Rémy Tremblay}

$\mathbf{L}$ e géographe Christian Morissonneau (1983) a été le premier à souligner l'importance de la Floride dans le contexte de l'Amérique française. Selon lui, il n'y a pas véritablement de sud au Québec; le Sud québécois, c'est la Floride. Et pour cause. Depuis près d'une vingtaine d'années, au-delà de 100000 Québécois se rassemblent en banlieue de Miami, ce que nous appellerons ici Floribec. Paradoxalement, malgré le caractère unique de Floribec au sein de la Franco-Amérique et toute l'attention que les médias lui portent, les chercheurs s'y sont très peu intéressés. Le but de cet article est justement de présenter brièvement, dans une perspective spatiotemporelle, la communauté ethnotouristique (une communauté ethnique qui repose sur le tourisme) de Floribec. Nous verrons également, à la lumière du modèle de cycle de vie des destinations touristiques de Butler (1980), que Floribec est maintenant en phase de déclin et ce, malgré la grande popularité qu'elle a connue dans les années 1980 et 1990.

Mais d'abord, qu'est-ce que Floribec? Il s'agit d'un espace touristique dans lequel la vie quotidienne s'anime autour de lieux structurants et d'institutions sociales, lesquels sont issus de rapports socio-spatiaux étroits entre les immigrants et les touristes de langue française et de culture québécoise ${ }^{2}$.

Mentionnons d'emblée que Floribec n'est pas unique. Par exemple, dans son étude sur les Britanniques en Espagne, O'Reilly (2000) montre une communauté qui s'ap- parente beaucoup à celle de Floribec. Les Finlandais ont, eux aussi, leur communauté ethno-touristique, comme l'a si bien présentée Timothy (2002) dans son étude sur la Petite Finlande de Lake Worth en Floride. Toutefois, ce qui distingue Floribec de ces autres communautés est qu'elle a été mise en place par un peuple linguistiquement et culturellement minoritaire en Amérique du Nord. Les Québécois ont réussi, avec succès, à se bâtir une communauté touristique en banlieue d'une des métropoles les plus dynamiques du continent.

\section{Les premiers signes}

D'après Dupont (1983), les Canadiens français commencent à immigrer en Floride dans les années 1930. Cette immigration est le fruit des investissements du gouvernement américain qui, à l'époque de la crise économique, décide d'améliorer la canalisation des marais du sud-est de la Floride (canal de quelques centaines de kilomètres appelé Intercoastal Waterway) et, du même coup, d'y développer l'infrastructure touristique. Des milliers d'Américains se rendront dans l'État du soleil pour travailler sur ce grand projet de construction, y compris des Franco-Américains de la NouvelleAngleterre, dont certains seront accompagnés de leurs cousins canadiens-français.

Une fois les travaux de construction terminés, plusieurs travailleurs d'origine canadienne-française s'établiront en permanence dans la région de Miami, plus précisément à Surfside, située dans l'est du comté de Dade (Miami métropolitain) sur les rives de l'Atlantique, ainsi qu'à North Miami. Après la Seconde Guerre mondia- le, on comptait 67000 familles canadiennes-françaises et franco-américaines dans l'État de la Floride (Dupont, 1983).

Ces nouveaux résidents permanents, surtout localisés à Surfside et à Sunny Isles (villes voisines) (figure 1), vont se recycler dans l'industrie touristique pour deux raisons simples: la Floride et Miami, en particulier, misent sur cette industrie et un nombre croissant de Canadiens français fortunés visitent cet État. Ce tourisme suscitera la première vague de migration massive de Québécois vers la Floride du Sud. On peut prétendre que cette première vague, qui s'est déroulée de 1946 à 1960, est à l'origine de Floribec comme nouveau foyer de l'Amérique française. C'est également à ce moment que démarre le cycle de vie touristique (modèle de Butler) de Floribec.

\section{La mise en place de la communauté floribécoise}

On assiste, de 1960 à 1970, à une seconde vague de migration québécoise vers la région de Miami, qui s'explique par deux phénomènes: la période de la Révolution tranquille au Québec, qui a favorisé une plus grande ouverture du Québec sur le monde, puis la diversification des loisirs pratiqués par les Québécois. Le tourisme de masse s'est développé. L'apparition des gros-porteurs, la construction des interstates américaines, le développement rapide des villes américaines de la Sun Belt, dont Miami, sont tous des facteurs qui ont largement contribué à l'explosion du tourisme en Floride et, par le fait même, à l'augmentation considérable de touristes québécois. Miami Beach et ses banlieues 


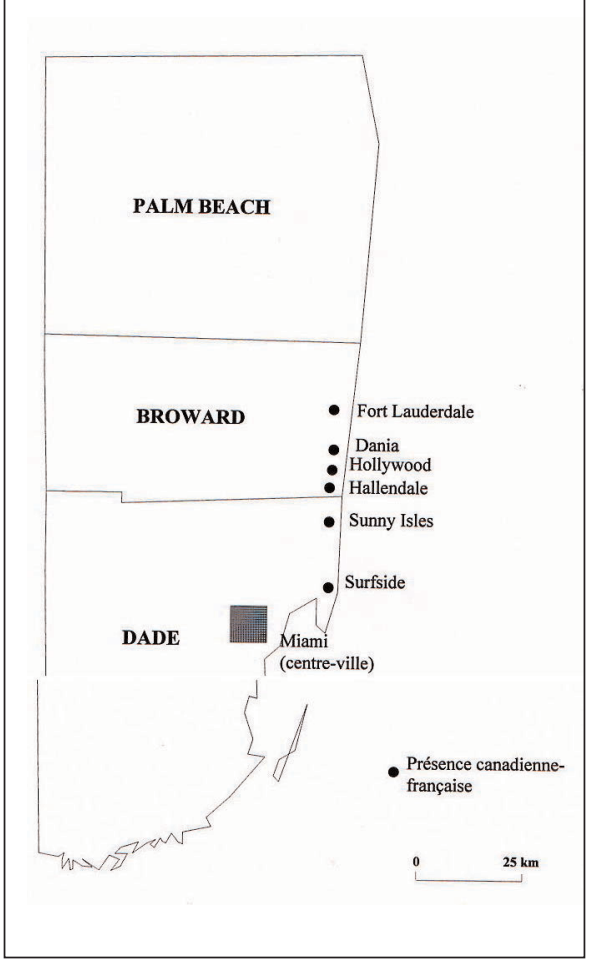

voisines de Surfside et de Sunny Isles deviendront les centres balnéaires privilégiés par ces touristes. C'est alors que des Floribécois commenceront à faire des affaires essentiellement axées sur le tourisme québécois. Ils posséderont surtout des motels, des restaurants, des bars et des dépanneurs, sachant très bien qu'il existe un réel désir de la part des touristes québécois de voir ce type d'entreprises leur offrir des services en français et, idéalement, en fonction de leurs propres besoins culturels (information, alimentation, habitation, etc.).

Ainsi, avec la mise sur pied de certains services offerts en français par des Québécois pour des Québécois, on observe de plus en plus dans les années 1970 une concentration de touristes et de résidents à Surfside et à Sunny Isles, notamment sur l'avenue Collins (à moins d'un kilomètre de l'Océan). Il devient alors évident que la destination touristique favorite des Québécois est non seulement accessible financièrement, elle l'est aussi sur le plan ethno-linguistique, car la barrière qu'est la langue anglaise n'y fait plus vraiment obstacle. Les hôtels Thunder- bird, Suez, Waikiki et Colonial sont connus de tous les Québécois familiers avec la Floride.

\section{Le déplacement de Floribec vers le nord}

Durant les années 1980, cet espace floribécois gravitant autour de Surfside et de Sunny Isles se déplacera. Miami, paradis du soleil, devient aussi le paradis des vices. Après la mafia dans les années 1920-1930, Miami devient un des centres internationaux du trafic de la drogue en plus d'être le théâtre de conflits raciaux majeurs (Boswell, 1991). Elle est couronnée «Capitale de l'Amérique latine», non seulement parce qu'elle devient la plaque tournante de l'Amérique latine avec sa centaine de banques latino-américaines, mais aussi parce qu'elle attire, dans son centre, des centaines de milliers de Cubains et autres Hispaniques. Cela cause un exode majeur des $\mathrm{WASP}^{3}$ vers les comtés de Broward et de Palm Beach (partie nord de la région de Miami/comté de Dade), et laisse toute la place voulue aux Hispaniques. Les touristes, y compris les Québécois, et les Floribécois suivront les WASP. Un semblable mouvement intra-urbain des populations correspond au modèle de sociologie urbaine d'invasionsuccession de l'École de Chicago.

\section{Les heures de gloire de Floribec}

Au début des années 1990, les espaces floribécois de Surfside et de Sunny Isles ont presque tous disparus. On a rasé les hôtels, les motels et les vieux édifices sans valeur architecturale, où se logeaient nombre de Québécois, pour y construire de luxueux condominiums postmodernes de plus de trente étages (souvent la propriété de Colombiens ). Cela explique en bonne partie pourquoi les villes voisines de Hallandale, de Hollywood et de Dania ont connu à cette période une expansion si importante auprès des immigrants et des touristes québécois.

Cette partie de la grande région de Miami est la plus au sud des refuges touristiques québécois abordables et ce, parce qu'il s'agit d'un des seuls endroits de la côte sud-est de la Floride à disposer de petits motels modestes, tant recherchés par les touristes de masse québécois, qui ont été épargnés des démolitions engendrées par l'étalement urbain. En fait, c'est dans cette partie de la Floride du sud que l'espace de la communauté ethno-touristique de Floribec prend racine et que sa vie quotidienne est la plus animée ${ }^{4}$ (figure 2).

Sur le plan de son organisation spatiale, Floribec prend la forme d'un «T», typique des districts d'affaires récréationnelles (DAR) de plusieurs autres stations balnéaires nord-américaines. On trouve ces DAR dans des villes axées exclusivement sur le tourisme, comme Atlantic City, Virginia Beach, Myrtle Beach et, plus près de nous culturellement et géographiquement, Old Orchard Beach et Hampton Beach. Le DAR floribécois, comme celui des autres stations balnéaires, dispose d'un « strip » linéaire parallèle à la plage, le Broadwalk, et d'une rue principale perpendiculaire à celui-ci, la rue Johnson. Sur ces deux artères vitales du DAR, on trouve la plupart de ce que les pionniers du concept de DAR décrivent comme «[a] seasonally oriented linear aggregation of restaurants, various specialty food stands, candy stores and a varied array of novelty and souvenirs shops that cater to visitor's leisurely shopping needs» (Stansfield et Rickert, 1970:219). Le long de la plage floribécoise se trouvent les plus importants lieux structurants (entre autres le Broadwalk et la scène de la rue Johnson) et institutions sociales (dont le restaurant Frenchie's Café). À l'extérieur du DAR, mais toujours dans la zone HollywoodDania-Hallandale, se trouvent une importante population touristique immigrante, d'origine québécoise ${ }^{5}$, de même que des institutions sociales floribécoises, telles que la Caisse populaire Desjardins, la Banque nationale (voir figure 3 ) et les bureaux du journal Le Soleil de la Floride. Au-delà de ce centre, c'est-à-dire à l'extérieur de la zone Hollywood-Dania- Hallandale, on trouve une périphérie où la population floribécoise, de même que les quelques points d'ancrage de la vie quotidienne, se diluent dans la culture anglo-saxonne. 


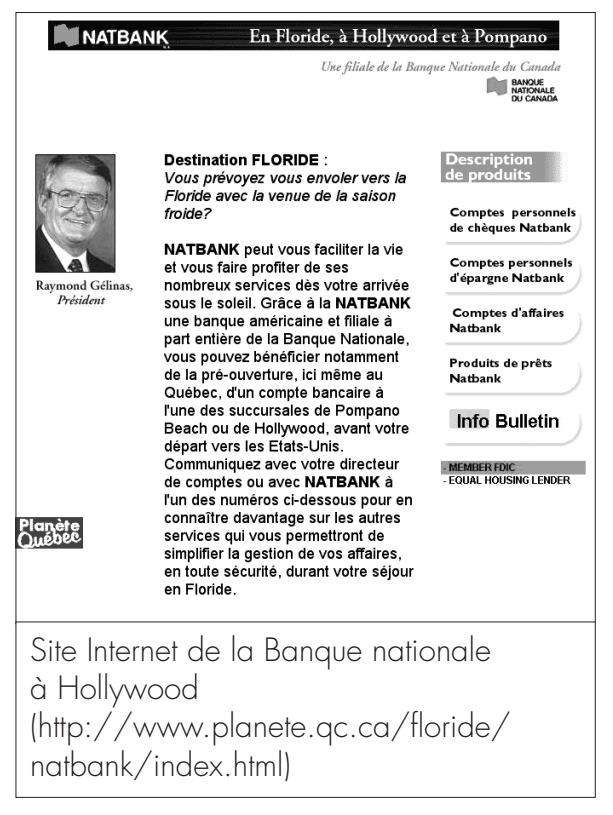

À ce DAR, ce centre et cette périphérie s'ajoute le Québec, car il fait partie intégrante de l'espace communautaire floribécois. L'apport saisonnier de milliers de touristes, l'accès facile aux journaux du Québec à Floribec, à la radio et aux principales émissions télévisées, font en sorte que Floribec garde des liens directs et constants avec le Québec. En fait, ces rapports touristiques et médiatiques avec le Québec constituent des institutions floribécoises à distance, lesquelles contribuent à étendre les bases socio-spatiales de la communauté. Floribec illustre donc parfaitement le concept de communauté transnationale, communauté qui tire profit des nouvelles technologies de l'information et des communications (NTIC) pour éviter l'isolement territorial.

Bref, au milieu des années 1990, les Québécois avaient réussi à se bâtir une communauté ethno-touristique comprenant de nombreux services, lieux structurants et institutions sociales, en banlieue d'une des villes américaines les plus en vogue.

\section{Le déclin de Floribec}

Depuis les cinq dernières années, Floribec a dramatiquement changé. Certes, les touristes québécois fréquentent toujours le sud-est floridien, mais la vie de langue française et de culture québécoise ne do- mine plus dans le DAR. En vérité, ce dernier s'éteint à un rythme accéléré ${ }^{6}$. Comment expliquer un tel déclin, qui correspond parfaitement au modèle de cycle de vie touristique de Butler? Selon nous, voici les principaux facteurs qui ont contribué à l'effritement de Floribec:

\section{L'étalement urbain de Miami}

La démolition des motels floribécois par de riches promoteurs immobiliers locaux et latino-américains ne s'est pas limitée aux villes de Surfside et de Sunny Isles. Faute de terrains disponibles, on frappe maintenant à la porte de Hollywood. À titre d'exemple, un immense complexe hôtelier de luxe a été construit à quelques kilomètres à peine du Broadwalk et on prévoit démolir des motels floribécois situés sur la plage de Hollywood pour faire place à des condominiums de luxe. Fort Lauderdale, juste au nord de Hollywood, est aussi en pleine effervescence et a vu pousser sur sa plage depuis deux ans quelques-uns des plus luxueux condominiums de la Floride. Hollywood est donc prise dans un étau et succombera à la pression d'ici les dix prochaines années. Une fois le DAR détruit, les Floribécois seront éparpillés et se regrouperont ça et là dans les parcs de maisons mobiles du comté de Broward.

\section{Des Floribécois moins «populaires»}

La classe socioprofessionnelle à laquelle appartiennent les Floribécois dérange la mairesse de Hollywood. Selon le modèle psychographique des touristes de Plog (1974), les Floribécois seraient psychocentriques, c'est-à-dire qu'il s'agirait de touristes de masse qui ne recherchent aucunement l'exotisme. Si, dans les années 1980, la mairesse appréciait les retombées économiques de ces derniers, elle s'est aperçue au fil des ans que, d'une part, les Floribécois projetaient une image peu reluisante de sa ville et, d'autre part, Hollywood aurait intérêt à emboîter le pas des villes voisines qui s'orientent vers l'élite touristique. En termes strictement économiques (dépenses quotidiennes par touriste, taxes foncières, etc.), Hollywood n'a rien à gagner en gardant les Floribécois chez elle. La mairesse a la ferme intention de redorer l'image touristique de sa ville, trop souvent victime de railleries de la part des médias locaux qui se moquent constamment des Floribécois. En fait, elle a déjà posé un premier geste en démolissant une des plus importantes institutions de Floribec: le Frenchie's Café, situé au coin du Broadwalk et de la rue Johnson, et les petits commerces voués à cette clientèle qui y étaient adjacents. Depuis, le DAR floribécois a perdu son âme. L'ambiance floribécoise si chère aux touristes est pratiquement disparue. Le seul véritable moment où les Floribécois reprennent le contrôle de ce coin subtropical est lors du CanadaFest, événement annuel regroupant sur le Broadwalk des commerçants floribécois et des chanteurs du Québec et attirant, d'après le Miami Herald, environ 100000 visiteurs. Les leaders économiques floribécois ont sans doute compris le message de la mairesse. Même si certains prétendent que tout va bien à Floribec, d'autres savent très bien que ses jours sont comptés.

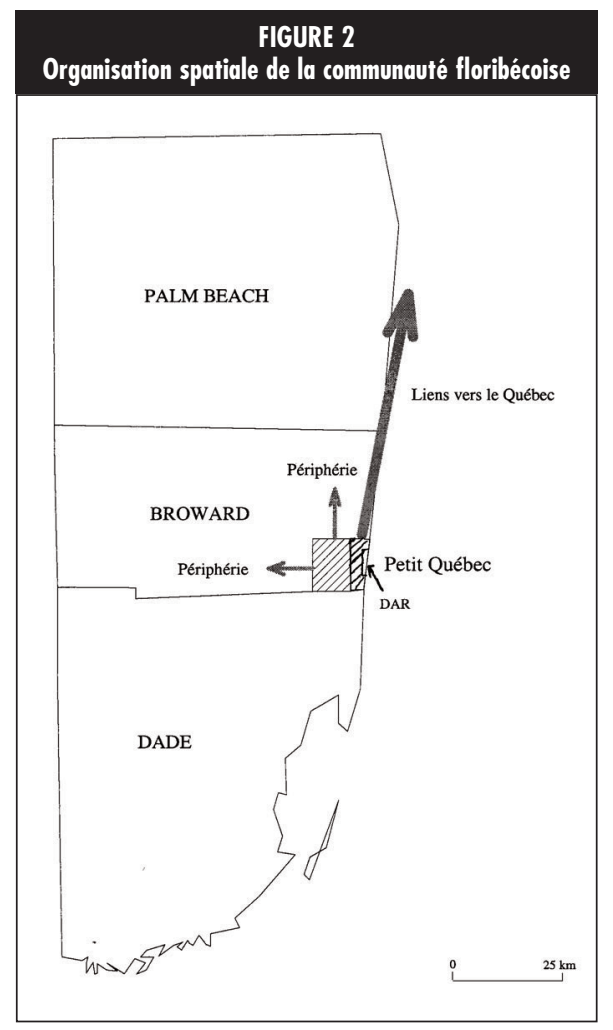




\section{Floribec et la concurrence}

Le dernier facteur qui nuit à la survie de Floribec est la multiplication des destinations à prix abordables dans les Antilles, les Caraïbes et en Amérique centrale. Considérant que les amants de la Floride du sud ne peuvent supporter des températures de moins de 25 degrés Celsius ${ }^{7}$ et que le dollar canadien a perdu de sa force par rapport à celui des États-Unis, ces nouvelles destinations pourraient faire du tort à l'économie de Floribec. En fait, il semblerait que la concurrence se fasse sentir puisque que Le Soleil de la Floride ne cesse de vanter les mérites de l'État du soleil dans ses pages. Les lecteurs considéreraient-ils la République dominicaine?

En somme, Floribec constitue un pan d'histoire du Québec et de la FrancoAmérique des plus fascinants. Même si l'étalement urbain de Miami, les plans de la mairesse de Hollywood et l'apparition de nouvelles destinations touristiques abordables feront probablement disparaître Floribec, elle permettra aux chercheurs de mieux nous éclairer sur la «vie et la mort» (pour paraphraser l'urbaniste Jane Jacobs ) d'une communauté ethno-touristique en banlieue d'une métropole américaine.

\section{Rémy Tremblay est docteur en} géographie de l'Université d'Ottawa. Il est présentement stagiaire postdoctoral à l'INRS-Urbanisation, Culture et Société, à Montréal.

\section{Notes}

1 L'auteur tient à remercier Anne Gilbert, géographe à l'Université d'Ottawa, pour ses conseils judicieux tout au long de ses études de doctorat. Comme il se doit, il est le seul responsable du contenu de cet article tiré de sa thèse.

2 Pour une discussion théorique sur Floribec comme communauté, lire Tremblay (2000a ; 2001 ; à paraître)

3 White Anglo-Saxon Protestant, personne de race blanche, d'origine anglo-saxonne et protestante.

4 Pour une analyse de l'organisation sociospatiale de Floribec et des nombreux lieux structurants et institutions sociales, voir Tremblay (2000b).

5 Si le US Census de 1990 dénombre près de 2000 Américains d'origine canadiennefrançaise vivant dans l'est de Hollywood, le nombre de touristes québécois est plus difficile à déterminer, faute de sources précises. L'association de résidents et de touristes québécois en Floride prétend qu'il y aurait, bon an mal an, quelque 300000 Québécois qui visiteraient le sud-est floridien.

6 Ces affirmations reposent sur les observations de terrain de l'auteur, lesquelles ont été rapportées par plusieurs médias écrits et télévisés québécois, canadiens-anglais et étatsuniens.

7 Nos recherches ont démontré que les touristes qui adoptent Floribec ne fréquentent pas la côte Ouest de la Floride (St. Petersburg, Clearwater, etc.) parque qu'ils considèrent qu'il y fait trop froid. Les Québécois qui visitent d'autres régions de la Floride ne fréquentent pas Floribec parce qu'ils n'apprécient pas les Québécois qui la visitent et qui l'habitent.

\section{Bibliographie}

Butler, Richard W. (1980), «The Concept of a Tourist Area Cycle of Evolution», Canadian Geographer, vol. 24, n 1, p. 5-12.

Boswell, Thomas (dir.) (1991), South Florida: The Winds of Change, Miami, Prepared for the Annual Conference of the Association of American Geographers.

Dupont, Louis (1983), «Le déplacement et l'implantation de Québécois en Floride», Vie française, 36 (10-11-12), p. 23-33.

Jacobs, Jane (1961), The Death and Life of Great American Cities, New York, Vintage, $458 \mathrm{p}$.

Morissonneau, Christian (1983), «Le peuple dit ingouvernable du pays sans bornes: mobilité et identité québécoise», dans Dean Louder et Éric Waddell (dir.), Du continent perdu à l'archipel retrouvé: le Québec et l'Amérique française, Sainte-Foy, Presses de l'Université Laval.

O'Reilley, Karen (2000), The British on the Costa Del Sol, London, Routledge.

Plog, Stanley (1974), «Why Destination Areas Rise and Fall in Popularity?», Cornell Hotel and Administration Quarterly, p. 55-58.

Stansfield, Charles, et John Rickert (1970), «The Recreational Business District», Journal of Leisure Studies, vol. 2, n 4, p. 213-225.
Timothy, Dallen (2002), «Tourism and the Growth of Tourism Ethnic Islands », dans Michael Hall et Allan Williams (dir), Tourism Migration, London, Routledge.

Tremblay, Rémy (à paraître), «Géographie, espace social et communauté floribécoise», Canadian Journal of Urban Research.

Tremblay, Rémy (2001), Floribec ou les Québécois en vacances, Montréal, INRS-urbanisation, culture et société.

Tremblay, Rémy (2000a), «Explorer la Floride canadienne-française», dans André Fauchon (dir), La francophonie panaméricaine: état des lieux, Winnipeg, Presses universitaires de SaintBoniface.

Tremblay, Rémy (2000b), Le concept de communauté en géographie vu à travers le Petit Québec de la Floride, Thèse de doctorat non publiée, Département de géographie, Université d'Ottawa. 